\title{
Medial Antebrachial Cutaneous Nerve Injury After Brachial Plexus Block: Two Case Reports
}

\author{
Mi Jin Jung, MD, Ha Young Byun, MD, Chang Hee Lee, MD, \\ Seung Won Moon, MD, Min-Kyun Oh, MD, Heesuk Shin, MD
}

Department of Rehabilitation Medicine, Gyeongsang National University Hospital, Jinju, Korea

\begin{abstract}
Medial antebrachial cutaneous (MABC) nerve injury associated with iatrogenic causes has been rarely reported. Local anesthesia may be implicated in the etiology of such injury, but has not been reported. Two patients with numbness and painful paresthesia over the medial aspect of the unilateral forearm were referred for electrodiagnostic study, which revealed MABC nerve lesion in each case. The highly selective nature of the MABC nerve injuries strongly suggested that they were the result of direct nerve injury by an injection needle during previous brachial plexus block procedures. Electrodiagnostic studies can be helpful in evaluating cases of sensory disturbance after local anesthesia. To our knowledge, these are the first documented cases of isolated MABC nerve injury following ultrasound-guided axillary brachial plexus block.
\end{abstract}

Keywords Peripheral nerve injuries, Local anesthesia, Electrodiagnosis

\section{INTRODUCTION}

Medial antebrachial cutaneous (MABC) neuropathy may occur due to iatrogenic causes, such as steroid injection, venipuncture, cubital tunnel surgery, and elbow arthroscopy $[1,2]$. Furthermore, although rare, it may also occur due to repetitive microtraumas, soft-tissue laceration, neuritis due to tuberculoid leprosy, or subcutaneous lipoma [3]. Axillary brachial plexus block is widelyused for finger and forearm surgery because it does not

Received March 4, 2013; Accepted June 12, 2013

Corresponding author: Heesuk Shin

Department of Rehabilitation Medicine, Gyeongsang National University Hospital, 79 Gangnam-ro, Jinju 660-702, Korea

Tel: +82-55-750-8252, Fax: +82-55-750-8255, E-mail: hsshin@nongae. gsnu.ac.kr

(c) This is an open-access article distributed under the terms of the Creative Commons Attribution Non-Commercial License (http://creativecommons. org/licenses/by-nc/3.0) which permits unrestricted noncommercial use, distribution, and reproduction in any medium, provided the original work is properly cited.

Copyright @ 2013 by Korean Academy of Rehabilitation Medicine cause complications, such as pneumothorax and phrenic nerve block that may occur in other brachial plexus blocks. In addition, it can be easily performed using a nerve stimulator or ultrasound guidance. The reported rate of neurological complications after axillary brachial plexus block is diverse, ranging from $0.2 \%$ to $19 \%$. These complications are generally temporary, and associated symptoms disappear without any particular treatment [4].

We report two cases of MABC nerve injuries after brachial plexus block. This is the first report of this type in the literature.

\section{CASE REPORTS}

\section{Case 1}

A 62-year-old female patient presented to the Department of Anesthesiology of this hospital with the chief complaints of 1-month duration of pain and numbness in her right medial antebrachial region. She was referred to the Department of Rehabilitation for electrodiagnos- 
tic examinations. According to the patient's past history, she had undergone right carpal tunnel release 8 months before she visited our hospital. However, this did not relieve the complaint of right finger numbness that she had before the surgery. Therefore, she had undergone the surgery again one month earlier. The symptoms in the medial antebrachial region occurred thereafter. During the surgery, she was anesthetized by axillary brachial plexus block using ultrasound guidance. First, in a supine position, her arm was externally rotated by $90^{\circ}$, her elbow joint was flexed by $90^{\circ}$, and her head was turned to face the side opposite to the surgery. The ultrasonic probe was placed on the axillary region to find the axillary artery, the surrounding structures were checked, and a $23-\mathrm{G}$ needle was inserted to inject a solution comprising a mixture of $2 \%$ lidocaine, $0.5 \%$ bupivacaine, and epinephrine $(1: 200,000)$ into the axillary sheath. Physical examinations revealed no muscle weakening but right thenar muscle atrophy. In sensory tests, the patient complained of decreased sensation and numbness in the right first, third, and fourth fingers, and in the medial antebrachial region. In particular, she reported that she suffered sleep disturbance due to the pain in the medial antebrachial region. The upper extremity deep tendon reflexes were all normal, but positive responses were obtained in the median nerve compression test. The symptoms were not accompanied by cervical and upper extremity pain, or decreased sensation. According to the electrodiagnostic examinations conducted 4 weeks after the onset of the symptom, the median and ulnar nerves were normal in motor nerve conduction studies. Sensory nerve conduction studies revealed a $4.10 \mathrm{~ms}$ delay in latency of the right median nerve and no response from the right MABC nerve (Table 1). Needle electromyography revealed no abnormal spontaneous activities in the abductor pollicis brevis muscle, which is innervated by the median nerve in the right-hand region.

Based on the results of the physical and electrodiagnostic examinations, the patient was diagnosed with right MABC nerve injury. Pregabalin $75 \mathrm{mg}$, clonazepam $0.5 \mathrm{mg}$, and tramadol $50 \mathrm{mg}$ were orally administered as drug treatment, and stellate ganglion block was performed. However, the patient's symptoms were not relieved. We decided to conduct outpatient follow-ups and periodic electrodiagnostic examinations. Eight weeks after the onset of the symptoms, the patient's pain was

Table 1. Results of nerve conduction studies of case 1

\begin{tabular}{ccccc}
\hline Nerve & Latency $(\mathbf{m s})$ & Amplitude $(\mathbf{m V})$ & Amplitude $(\boldsymbol{\mu V})$ & Velocity $(\mathbf{m} / \mathbf{s})$ \\
\hline Motor (R/L) & & & & \\
\hline Median & $3.85 / 3.65$ & $10.9 / 9.6$ & - & $55.3 / 53.8$ \\
\hline Ulnar & $2.25 / 2.20$ & $11.6 / 10.3$ & - & $60.9 / 65.7$ \\
Sensory (R/L) & & & & \\
Median & $4.10 / 3.65$ & - & $39.8 / 42.2$ & - \\
Ulnar & $2.95 / 3.05$ & - & $29.3 / 19.9$ & - \\
MABCN & Not evoked/2.4 & - & Not evoked/5.7 & - \\
\hline
\end{tabular}

$\mathrm{R}$, right; L, left; MABCN, medial antebrachial cutaneous nerve.

Table 2. Results of nerve conduction studies of case 2

\begin{tabular}{ccccc}
\hline Nerve & Latency $(\mathbf{m s})$ & Amplitude $(\mathbf{m V})$ & Amplitude $(\boldsymbol{\mu V})$ & Velocity $(\mathbf{m} / \mathbf{s})$ \\
\hline Motor (R/L) & & & & \\
\hline Median & $3.20 / 3.95$ & $12.9 / 14.4$ & - & $55.3 / 56.3$ \\
Ulnar & $2.45 / 2.80$ & $13.4 / 14.2$ & - & $56.0 / 55.4$ \\
Sensory (R/L) & & & & \\
Median & $3.25 / 3.60$ & - & $43.8 / 50.9$ & - \\
\hline Ulnar & $2.95 / 3.05$ & - & $61.4 / 51.3$ & - \\
MABCN & $1.80 / 1.95$ & - & $8.6 / 10.6$ & - \\
\hline
\end{tabular}

$\mathrm{R}$, right; L, left; MABCN, medial antebrachial cutaneous nerve. 
relieved slightly. In follow-up sensory nerve conduction study of the right MABC nerve, the latency on the right side was delayed by $5.00 \mathrm{~ms}$ compared to $2.50 \mathrm{~ms}$ on the unaffected left side, and the amplitude on the right side showed an abnormally reduced value of $2.9 \mu \mathrm{V}$ compared to $10.1 \mu \mathrm{V}$ on the left side. In addition, in the somatosensory evoked potential, the N20 latency of the right MABC nerve was delayed by $22.60 \mathrm{~ms}$ compared to $17.70 \mathrm{~ms}$ on the left unaffected side. The N20-P25 amplitude on the right side was $1.30 \mu \mathrm{V}$, showing a reduction of at least $50 \%$ compared to the left side $(3.85 \mu \mathrm{V})$ (Table 3$)$.

\section{Case 2}

A 30-year-old female patient was treated in the Department of Anesthesiology of this hospital for pain and numbness in the right medial antebrachial region that had begun 8 weeks earlier. She was referred to the Department of Rehabilitation for electrodiagnostic examinations. She had been receiving conservative treatment for an enchondroma following a fracture of the third proximal phalanx of her right hand suffered 6 months earlier during a fall. She had undergone curettage and a bone graft under ultrasound-guided axillary brachial plexus block 8 weeks prior to the present visit. She experienced numbness in the right medial antebrachial region immediately after the surgery. Her past medical history was unremarkable. Physical examinations revealed no particular finding other than decreased sensation and numbness of the right medial antebrachial region. According to the electrodiagnostic examinations conducted 8 weeks after onset, the median nerve and the ulnar nerve were both normal in motor nerve conduction studies. In sensory nerve conduction studies, the latency of the right median nerve was $1.80 \mathrm{~ms}$ and $1.95 \mathrm{~ms}$ on

Table 3. Results of median antebrachial cutaneous somatosensory evoked potentials

\begin{tabular}{lcc}
\hline & $\begin{array}{c}\text { Latency (ms) } \\
\text { C3'/C4' (N20) }\end{array}$ & $\begin{array}{c}\text { Amplitude }(\boldsymbol{\mu V}) \\
\text { N20-P25 }\end{array}$ \\
\hline Case 1 (R/L) & $22.60 / 17.70$ & $1.30 / 3.85$ \\
Case 2 (R/L) & $16.80 / 15.90$ & $1.33 / 1.56$ \\
Reference data & & \\
To peak & $17.4 \pm 1.2$ & - \\
\hline R/L difference & $0.5 \pm 0.4$ & - \\
\hline
\end{tabular}

$\mathrm{R}$, right; $\mathrm{L}$, left. the unaffected left side. The amplitude on the right side was $8.6 \mu \mathrm{V}$, showing a slight reduction compared to the left side $(10.6 \mu \mathrm{V})$, although the difference was not clinically significant (Table 2). In needle electromyography, no abnormal spontaneous activities were observed in the muscles innervated by the median nerve or in the ulnar nerve of the right-hand region.

Based on the results of the physical examinations and the electrodiagnostic examinations, right MABC nerve injury was suspected. Pregabalin $75 \mathrm{mg}$, clonazepam $0.5 \mathrm{mg}$, and tramadol $50 \mathrm{mg}$ were orally administered, and stellate ganglion block was performed. However, the treatment did not relieve the patient's symptoms. In follow-up sensory nerve conduction study of the right MABC nerve 13 weeks after the onset of the symptoms, the amplitude had increased slightly to $10.6 \mu \mathrm{V}$, and the patient reported that the numbness and pain in the right medial antebrachial region were considerably relieved. In the somatosensory evoked potential, the N20 latency of the MABC nerve on the right side was $16.80 \mathrm{~ms}$ and 15.90 ms on the left side. The N20-P25 amplitude on the right side was $1.30 \mu \mathrm{V}$ and $1.56 \mu \mathrm{V}$ on the left side (Table 3 ).

\section{DISCUSSION}

The MABC nerve arises from the medial cord or the inferior trunk of the brachial plexus and runs together with the median nerve, ulnar nerve, brachial artery, and basilic vein on the upper arm (Fig. 1). It penetrates the brachial fascia, together with the basilic vein, $10 \mathrm{~cm}$ above the medial epicondyle, running toward the distal region and ramifying the anterior and the posterior branches to supply the medial forearm senses [5]. MABC neuropathy may be the result of diverse iatrogenic causes including repetitive microtraumas or subcutaneous lipoma. In particular, due to the superficial location in the cubital fossa region, venipuncture may damage the nerve [1-3]. However, no MABC nerve injury has been reported in relation to local anesthesia. As the MABC nerve is purely a sensory nerve and does not affect the hand, in many cases patients do not complain of severe discomfort even when this nerve has been injured. Furthermore, the MABC nerve is not commonly examined in electrodiagnostic examinations. Therefore, MABC neuropathy is rarely diagnosed.

Nerve injuries related to peripheral nerve block are 


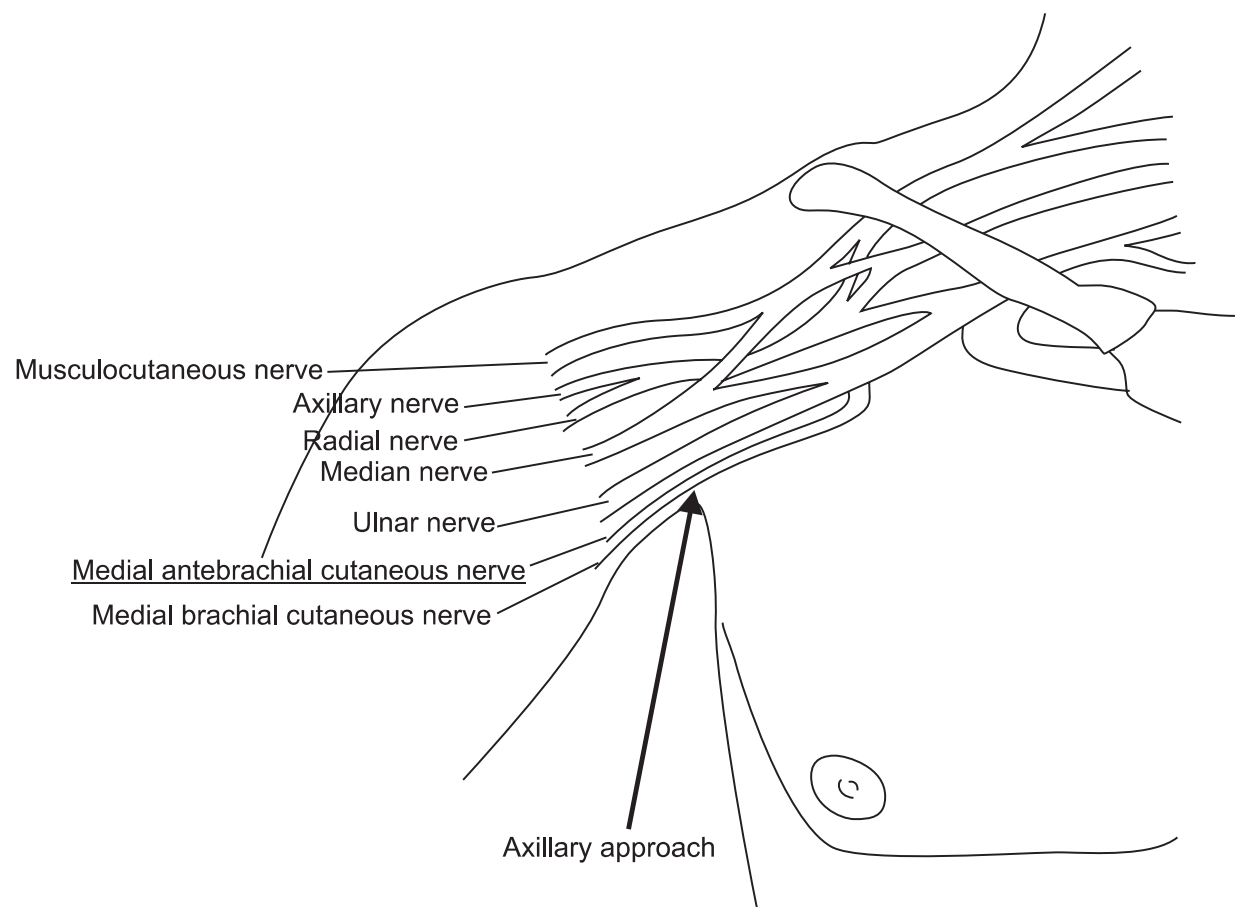

Fig. 1. Diagram of the medial antebrachial cutaneous nerve. most frequently reported in the ulnar nerve, followed by the brachial plexus, lumboiliac nerve, and spinal nerves in order of prevalence [6]. These frequencies are related to their anatomical locations. As the ulnar nerve runs through superficial paths on the medial side of the elbow, it can be easily compressed between the edge of the operating table and the medial epicondyle. Nerve injuries occur due to compression and to nerve extension or ischemia. The patient's position during surgery, previous nerve injuries, direct trauma during surgery, vascular catheter insertion, procedures for nerve block, and the use of tourniquets may cause nerve injuries [7].

In the two present cases, the patients' arms were externally rotated by $90^{\circ}$. Thus, the nerve was not likely to have been excessively extended. It is also unlikely that the MABC nerve was extended. Although the first case experienced numbness in the fingers of her right hand due to carpal tunnel syndrome, this was not related to the existing neuropathy because she experienced pain and numbness in the right medial antebrachial region after the nerve block. In the second case, the patient did not complain of any symptoms that might suggest neuropathy and had not been diagnosed with any nerve injuries. Furthermore, the possibility of direct injuries of the MABC nerve due to surgery could be excluded because the surgery sites were below the wrist in both cases. The possibility of nerve injuries due to vascular catheter insertion or drug administration could also be ruled out because the vascular catheters were inserted into the left upper arm in both cases. Tourniquets are used for periodic blood pressure measurements and to reduce hemorrhage during surgery. Nerve injuries related to the use of tourniquets are related to the pressure applied to the tourniquet and the time of application of the tourniquets. Makitie and Teravainen [8] advised that applying tourniquet pressure in the range of $250-500 \mathrm{mmHg}$ would not cause nerve conduction block, and Fanelli et al. [9] reported that pressure exceeding $350 \mathrm{mmHg}$ might cause nerve conduction block. The possibility of nerve injuries due to the application of excessive pressure to tourniquets was low in the present cases, as the tourniquets were applied for less than 1 hour at a pressure of $250 \mathrm{mmHg}$ in both cases.

In addition to direct injuries, isolated injuries of the MABC nerve due to repetitive trauma of the elbow joint region have been reported. Richards and Regan [1] reported a case of MABC nerve injury occurring immediately after injecting steroids into the medial epicondyle in a patient who complained of chronic elbow pain due to medial epicondylitis. Asheghan et al. [2] reported a case of direct isolated MABC nerve injury due to needles used in the process of venipuncture. Yildiz and Ardic [3] 
reported a case of isolated MABC nerve injury after microtraumas due to repetitive upper extremity activities [3]. In the latter case, the isolated injury occurred because the elbow joint was continuously flexed and extended in the process of the shaking of a rug.

Depending on the region to be anesthetized, brachial plexus block can be performed using interscalene, supraclavicular, infraclavicular, or axillary approaches. The anesthesia was performed through an axillary approach in the present cases. In both cases, the anesthetic was injected by inserting a needle into the sheath during axillary brachial plexus block. The fact that the injuries occurred only in the MABC nerve and that paresthesia was reported during the process of inserting the needles suggest that the injuries were caused by direct injury of the nerves by the needles (Fig. 2). Needles can injure the nerves because anatomically the MABC nerve branches from the medial cord or the inferior trunk of the brachial plexus, which are more proximal than the region through which the needles are inserted during brachial plexus block using an axillary approach. In addition, although the locations of major structures, such as the axillary artery, median nerve, radial nerve, and ulnar nerve, are identified during anesthesia, the MABC nerve, which is relatively more superficially located on the skin, is overlooked in many cases in the process of inserting the needle. Even purely sensory nerve injuries should not be considered insignificant if the patient complains of discomfort in daily living to the extent that the pain causes sleep disturbance or depression. Therefore, efforts should be made to prevent MABC nerve injuries during brachial plexus block. If the patient complains of paresthesia or pain in the medial antebrachial region after surgery, the possibility of MABC nerve injuries should be noted, and the injuries should be diagnosed early and treated appropriately. If these nerve injuries occur frequently, studies to understand the pathway and the location of this nerve will be necessary.

In the present cases, despite identifying the locations of various structures using ultrasound prior to the injection of the anesthetic and the insertion of the needles close to the nerve to be blocked, unexpected injuries occurred. Therefore, appropriate patients should be selected, and the patients should be assessed before surgery by clinicians with sufficient anatomical knowledge of the target region. In addition, needles known not to damage the nerves to any great extent should be selected, and the target region should be approached carefully. Moreover, attention should be paid when the anesthetic is injected into the nerves, and the necessary minimum concentration of local anesthetic should be used. Finally, the position of the patient's body during surgery and the tourniquet pressure applied should be carefully observed.

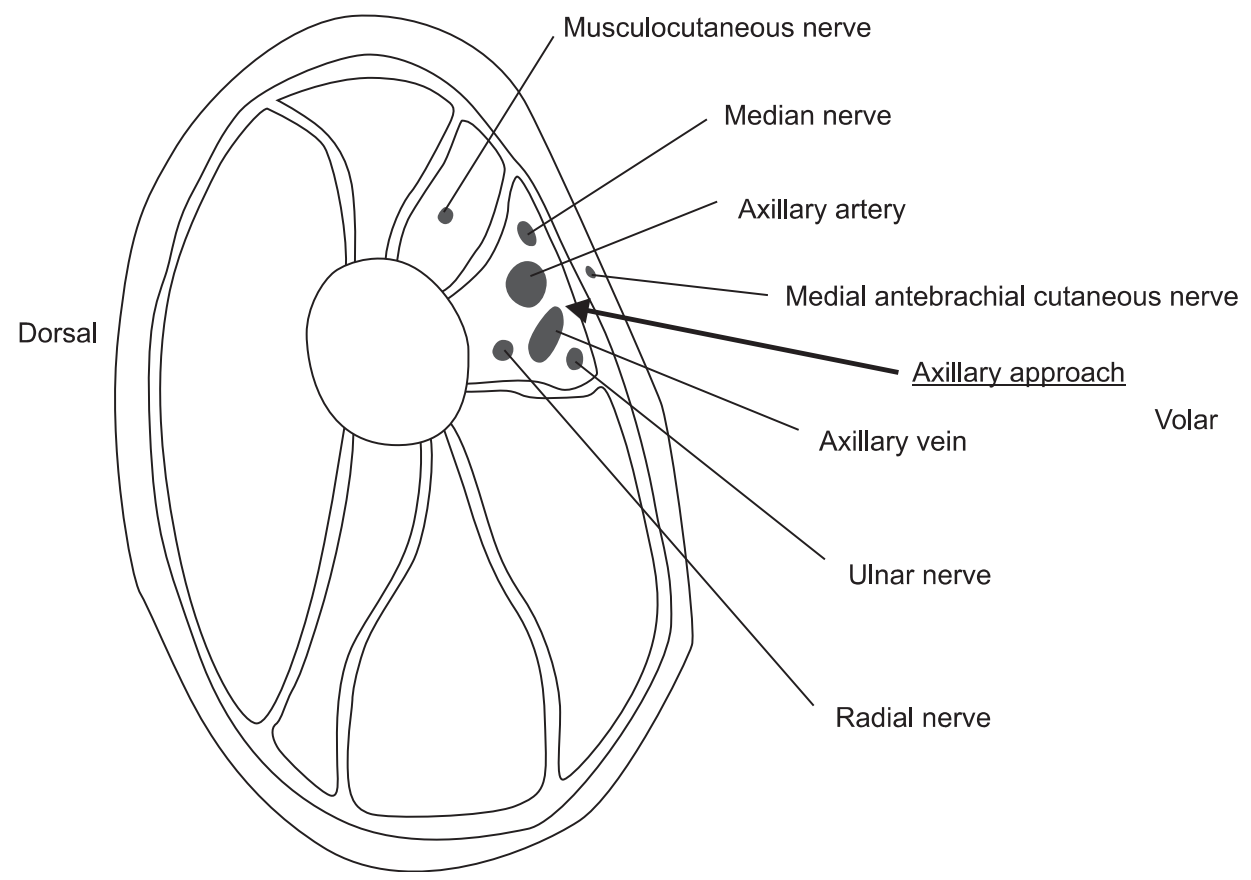

Fig. 2. Axillary approach, showing relationships of the nerves to the artery. 


\section{CONFLICT OF INTEREST}

No potential conflict of interest relevant to this article was reported.

\section{REFERENCES}

1. Richards RR, Regan WD. Medial epicondylitis caused by injury to the medial antebrachial cutaneous nerve: a case report. Can J Surg 1989;32:366-7, 369.

2. Asheghan M, Khatibi A, Holisaz MT. Paresthesia and forearm pain after phlebotomy due to medial antebrachial cutaneous nerve injury. J Brachial Plex Peripher Nerve Inj 2011;6:5.

3. Yildiz N, Ardic F. A rare cause of forearm pain: anterior branch of the medial antebrachial cutaneous nerve injury: a case report. J Brachial Plex Peripher Nerve Inj 2008;3:10.

4. Horlocker TT, Kufner RP, Bishop AT, Maxson PM, Schroeder DR. The risk of persistent paresthesia is not increased with repeated axillary block. Anesth Analg
1999;88:382-7.

5. Thallaj A, Marhofer P, Kettner SC, Al-Majed M, AlAhaideb A, Moriggl B. High-resolution ultrasound accurately identifies the medial antebrachial cutaneous nerve at the midarm level: a clinical anatomic study. Reg Anesth Pain Med 2011;36:499-501.

6. Sandhu K, Dash HH. Anaesthesia related neurological complications. Indian J Anaesth 2004;48:439-45.

7. Hebl JR, Horlocker TT, Sorenson EJ, Schroeder DR. Regional anesthesia does not increase the risk of postoperative neuropathy in patients undergoing ulnar nerve transposition. Anesth Analg 2001;93:1606-11.

8. Makitie J, Teravainen H. Peripheral nerve injury and recovery after temporary ischemia. Acta Neuropathol 1977;37:55-63.

9. Fanelli G, Casati A, Garancini P, Torri G. Nerve stimulator and multiple injection technique for upper and lower limb blockade: failure rate, patient acceptance, and neurologic complications. Study Group on Regional Anesthesia. Anesth Analg 1999;88:847-52. 